\title{
CREM: A TRANSCRIPTIONAL MASTER SWITCH DURING THE SPERMATOGENESIS DIFFERENTIATION PROGRAM
}

\author{
François Nantel and Paolo Sassone-Corsi ${ }^{1}$ \\ Institut de Génétique et de Biologie Moléculaire et Cellulaire, 1 rue Laurent Fries, BP 163, ILLKIRCH Cedex, C.U. \\ de Strasbourg, France
}

\section{TABLE OF CONTENTS}

1. Abstract

2. Introduction

3. Hormonal control of testicular function: The cAMP pathway

4. The CREM gene: Regulation and function in germ cells

5. CREM knockout mice

6. Conclusions and perspectives

7. Acknowledgments

8. References

\section{ABSTRACT}

Spermatogenesis is a complex differentiation process under the hormonal control of the hypothalamic-pituitary axis. The CREM gene encodes activators and repressors of cAMP-mediated gene transcription. The transcript corresponding to the activator isoform CREM $\tau$ is found at high levels in pachytene spermatocytes onwards. The CREM $\tau$ protein, however, is present only in post-meiotic spermatids where it activates the transcription of testis-specific genes, such as the protamines and transition proteins. Mice in which the CREM gene has been inactivated by homologous recombination have been generated. Homozygous male mutant mice are sterile and produce no spermatozoa. Histological analysis of the seminiferous tubules reveal a complete arrest of spermatogenesis at the first step of spermiogenesis. CREM deficiency results in the lack of post-meiotic gene expression and a ten-fold increase in apoptotic germ cells. These results demonstrate the essential role of CREM in spermatogenesis and are reminiscent of some cases of male infertility.

\section{INTRODUCTION}

Spermatogenesis is a complex differentiation process during which diploid germ cells differentiate into haploid spermatozoa. This process involves multiple biochemical and structural changes and requires the activation of a large array of

Received: 8/17/96 Accepted 8/21/96

${ }^{1}$ To whom correspondence should be addressed, at Institut de Génétique et de Biologie Moléculaire et Cellulaire, 1 rue Laurent Fries, BP 163, ILLKIRCH Cedex, C.U. de Strasbourg, France, Tel \#: (+33) 88 6534 06, FAX \#: (+33) 886532 46, E-mail: paolosc@titus.u-strabg.fr genes. Spermatogenesis is under the tight control of the hypothalamic-pituitary axis and requires the coordinate action of multiple hormones (1). These hormonal signals are converted into long-term changes in gene expression. The cyclic AMP (cAMP) signaling pathway plays a critical role in this differentiation cascade and recent research has identified transcription factor CREM (cyclic AMP responsive element modulator) as a master switch in cAMP-dependent gene transcription in the testis.

\section{HORMONAL CONTROL OF TESTICULAR FUNCTION: THE cAMP PATHWAY}

The gonadotropin releasing hormone (GnRH) released by the hypothalamus stimulates the secretion of follicule stimulating hormone (FSH) and luteinizing hormones $(\mathrm{LH})$ from the gonadotroph cells of the anterior pituitary (1-3). Receptors for FSH and LH are present on somatic Leydig and Sertoli cells, respectively, which have the function of transmitting the hormonal stimulus to the germ cells $(4,5)$. The cAMP pathway plays a critical role in this hormonal cascade. When binding of FSH and LH to their respective receptors occurs, it activates their coupling to Gs proteins and stimulates the enzyme adenylyl cyclase which catalyses the conversion of ATP to cAMP. Elevations in intracellular levels of cAMP stimulate the protein kinase A (PKA) which then phosphorylates several substrate proteins. Among the targets of PKA are transcription factors that recognize the cAMP-responsive element (CRE) sequence located on the promoter of many genes. Phosphorylation of these CRE-binding proteins by PKA activates them and drives cAMP-dependent gene expression (6). 


\section{THE CREM GENE: REGULATION AND FUNCTION IN GERM CELLS}

The CREM gene presents many remarkable characteristics. By alternative splicing, CREM isoforms may contain one of two distinct, alternative, DNA binding domains which confer distinct affinities for CREs $(7,8)$. In contrast to CREB which encode exclusively activators of gene transcription, the CREM gene may encode both activators $(\tau)$ and repressors $(\alpha, \beta, \gamma)(8,9)$. Moreover, CREM has a tissue- and cell-specific pattern of expression since it is found at high levels in the testis and neuroendocrine tissues $(7,10)$. Finally, CREM is an early response gene, a property conferred by an intronic promoter that is strongly inducible by cAMP (11). This internal promoter (P2) is located upstream from the DNA binding domains and drives the expression of ICER (Inducible cAMP early repressor), a powerful repressor of the cAMP-induced transcription that is the smallest transcription factor know to this day. The expression of the $\mathrm{P} 2$ promoter occurs through activation of four CRE sequences which are organized in tandem within the promoter. Following the induction of the ICER protein, this inhibit its own transcription by binding the four CRE sequences (11). Thus, there is a CREM negative autoregulatory loop, which ensures various cycles of transcriptional inducibility (11).

The CREM gene is highly expressed in the adult testis (10). Levels of CREM transcripts are low in prepubertal testis and only the repressor isoforms $(\alpha, \beta)$ are detected. However, during puberty, transcripts encoding the activator form CREM $\tau$ accumulate to high levels in pachytene spermatocytes and spermatids (10). Interestingly, the CREM $\tau$ protein is not detected in spermatocytes but only in haploid spermatids (12). Many haploid genes have been identified as potential CREM target genes since they contain CREs in their promoters. The genes encoding RT7, transition protein 1 and calspermin have all been shown to be activated by CREM $\tau$ (1214).

The switch in CREM expression pattern during spermatogenesis is under the control of the gonadotropin FSH (15). Surgical removal of the pituitary gland from adult rats leads to loss of $\operatorname{CREM} \tau$ expression in the testis and hypophysectomization of prepubertal rats prevents the developmental switch in CREM expression (15). The physiological role of pituitary hormones on CREM expression was also studied in the hamster, a seasonal breeder characterized by testicular atrophy and spermatogenic arrest during winter. Hamsters kept under conditions of short photoperiod (SP) show a reduction in serum concentrations of $\mathrm{FSH}, \mathrm{LH}$ and prolactin, accompanied by testicular atrophy and absence of CREM expression. Hormonal levels and CREM expression are restored when the animals are placed under long photoperiod (LP). Injection of FSH, but not LH or prolactin, in hamsters kept under SP increases CREM $\tau$ expression to levels found in sexually active animals (15). This observation clearly indicated that FSH is the hormone regulating CREM $\tau$ expression. The molecular mechanism by which FSH activates CREM $\tau$ expression in germ cell is under investigation. The FSH receptor is located on somatic Sertoli cell and not on the germ cells. During their developmental process, the germ cells are kept in intimate contact with the somatic Sertoli cells. It has been suggested that a paracrine factor, possibly a peptide, is released by the Sertoli cells following FSH stimulation and that this factor is responsible for the switch in CREM expression. Nuclear run-on experiments show that the increased CREM $\tau$ expression in germ cell is not associated with an increase in the rate of transcription initiation of the gene. Instead, the increase in CREM $\tau$ is characterized by the use of an alternative polyadenylation site, located in close proximity to the stop codon, that excludes 9 of the 10 AUUUA mRNA destabilization elements found on the 3 ' untranslated region of the CREM gene (15).

Recently, it has been shown that CREM may be implicated in the regulation of Sertoli cell function. In cultured primary Sertoli cells, FSH induces an increase in cAMP levels which results in the activation of the P2 promoter of CREM and then in an increase in the expression of ICER (16). Interestingly, the kinetics of ICER expression correlate with the transcriptional down-regulation of the FHS receptor. Given that ICER is a potent transcriptional repressor and that the FSH receptor gene has a CRE element in its promoter, it was postulated that ICER could be implicated in the reduction in FSH receptor gene expression. DNAbinding and expression studies have demonstrated that ICER is involved in the long-term desensitization of the FSH-receptor in Sertoli cells (16).

\section{CREM KNOCKOUT MICE}

The physiological role of CREM has been addressed with the development of mice in which the CREM gene is disrupted by homologous recombination $(17,18)$. Heterozygous mutant mice appeared normal and healthy although there was a slight reduction in the average litter size. Analysis of sperm from heterozygous male mice show a $46 \%$ reduction in sperm number, a $35 \%$ decrease in the ratio of motile spermatozoa and a two-fold increase in the proportion of spermatozoa with aberrant structures. 

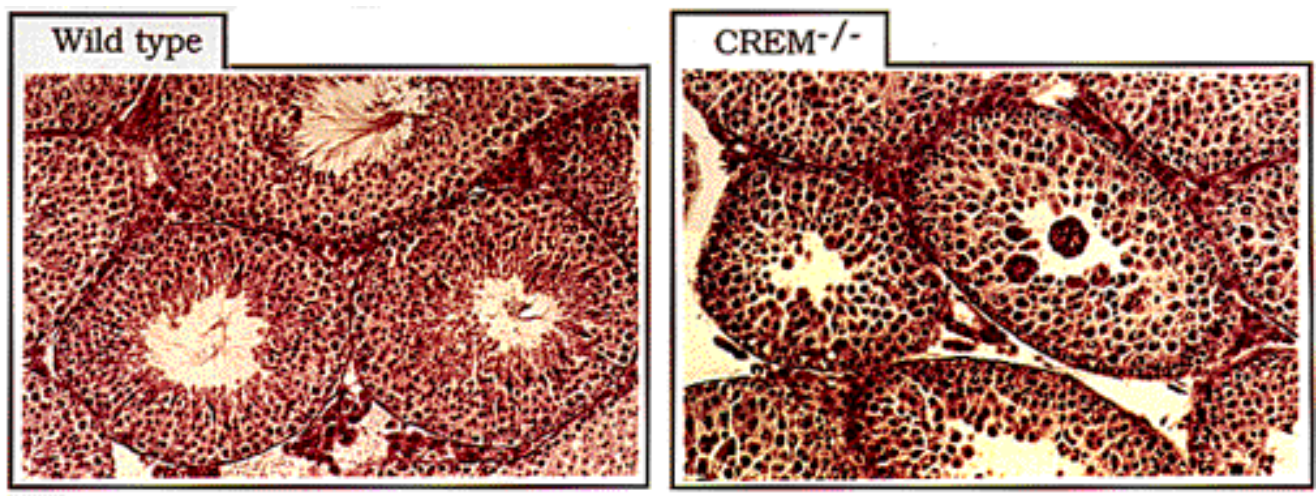

Figure 1: Histological analysis of testis from 8 weeks-old wild-type (left) and CREM knockout mice (right). Note the absence of developing spermatids and spermatozoa and the presence of multi-nucleated giant cells in the mutant animal.

Homozygous CREM mice appear healthy and present no obvious physical aberrations or weight loss. While the homozygous females are fertile, the males however are sterile. Testis weight in homozygous mice is $25 \%$ less than those found in wild-type animals and the seminal fluid was completely devoid of spermatozoa. Histological analysis of seminiferous tubules show that spermatogenesis in CREMdeficient mice is interrupted at the very early spermatid stage (Figure 1). Elongating spermatid and spermatozoa are completely absent, while premeiotic germ cells and somatic Sertoli and Leydig cells appear normal. In the testis from CREM-mutant mice, there is a ten-fold increase in multi-nucleated giant cells undergoing apoptosis. Analysis of gene expression in the testis shows that expression of postmeiotic genes is decreased in the CREM-deficient mice. These include genes encoding the protamines, transition proteins and RT7. Moreover, transcripts for Krox-20, Krox-24 and calspermin genes are also absent in testis from heterozygous animals.

These results indicate that CREM is essential for the differentiation program of spermiogenesis. The absence of CREM prevents the expression of many haploid-specific genes and the germ cells go into apoptosis following their meiotic division. The observation that FSH and testosterone levels are not reduced in CREM-mutant mice (18) suggests that these animals may constitute tools for the study of male infertility with normal gonadotropin level.

\section{CONCLUSIONS AND PERSPECTIVES}

In the five years that followed its isolation, the transcription factor CREM has been shown to be one of the key genes implicated in the regulation of the spermatogenesis differentiation cascade. Other mutant mice with testicular phenotype similar to the one found in CREM-mutant animals have been described $(19,20)$. Genes encoding the apoptosisrelated protein Bax (19) and the chaperone Hsp70-2 (20) are either implicated in the CREM-mediated differentiation cascade or are regulating the same or an alternative apoptosis pathway. Biochemical experiments and the development of double-knockout animals may help to address these questions.

The physiological consequences of CREM ablation have not been fully investigated especially in view of the complex expression pattern of this gene in neuroendocrine cells. Detailed analysis of endocrine and circadian alterations in the CREMmutant mice are presently underway in our laboratory.

\section{ACKNOWLEDGMENTS}

We would like to thank all members of our laboratory; L. Monaco, C. Mazzuchelli, E. Zazopoulos, E. Lalli, K. Tamai, M. Lamas, D. DeCesare, L. Penna, E. Heitz and N.S. Foulkes for help and discussion. FN is the recipient of a fellowship from the Medical Research Council of Canada. This work was supported by grants from CNRS, INSERM, CHUR, FRM, the Association pour la Recherche sur le Cancer and Rhône-Poulenc Rorer.

\section{REFERENCES}

1. J.D. Veldhuis: The hypothalamic-pituitarytesticular axis. In: Reproductive endocrinology. Eds: S.S.C. Yen \& R.B. Jaffe, W.B. Saunders Co., Philadelphia, 409-459, (1991)

2. E. Steinberger: Hormonal control of mammalian spermatogenesis. Physiol Rev 51, 1-22, (1971) 
3. A.J. Lostroh: Hormonal control of spermatogenesis. In Regulation mechanisms of male reproductive physiology. Eds: C.H. Spilman, T.J. Lobl \& K.T. Kirton, Excerpta Medica, Amsterdam, 13-23, (1976)

4. R.Y. Moore: Neuroendocrine regulation of reproduction. In Reproductive endocrinology. Eds: S.S.C. Yen \& R.B. Jaffe, W.B. Saunders Co., Philadelphia, 335-378, (1978)

5. R.J. Santen: The testis. In Endocrinology and metabolism. Eds: P. Felix, J.D. Baxter, A.E. Broadus \& L.A. Frohman, Mc Graw Hill Co, New-York, 821905, (1987)

6. P. Sassone-Corsi: Transcription factors responsive to cAMP. Annu Rev Cell Dev Biol 11, 355-377, (1995)

7. N.S. Foulkes, E. Borrelli \& P. Sassone-Corsi: CREM gene: use of alternative DNA binding domains generates multiple antagonists of cAMP-induced transcription. Cell 64, 739-749, (1991)

8. B.M. Laoide, N.S. Foulkes, F. Schlotter, P. Sassone-Corsi: The functional versatility of CREM is determined by its modular structure. EMBO J 12, 1179-1191, (1993)

9. V. Delmas, B.M. Laoide, D. Masquillier, R.P. deGroot, N.S. Foulkes \& P. Sassone-Corsi: Alternative usage of initiation codons in mRNA encoding the cAMP-responsive-element modulator (CREM) generates regulators with opposite functions. Proc Natl Acad Sci USA 89, 4226-4230, (1992)

10. N.S. Foulkes, B. Mellström, E. Benusiglio \& P. Sassone-Corsi: Developmental switch of CREM function during spermatogenesis: from antagonist to transcriptional activator. Nature 355, 80-84, (1992)

11. C.A. Molina, N.S. Foulkes, E. Lalli \& P. Sassone-Corsi: Inducibility and negative autoregulation of CREM: an alternative promoter directs the expression of ICER, an early response repressor. Cell 75, 875-886, (1993)

12. V. Delmas, F. van der Hoorn, B. Mellström, B Jégou \& P. Sassone-Corsi: Induction of CREM activator proteins in spermatids: downstream targets and implications for haploid germ cell differentiation. Mol Endocrinol 7, 1502-1514, (1993)

13. M.K. Kistler, P. Sassone-Corsi \& S.W. Kistler: Identification of a functional cAMP-response element in the 5'-flanking region of the gene for transition protein 1 (TP1), a basic chromosomal protein of mammalian spermatids. Biol Reprod 51, 1322-1329, (1994)
14. Z. Sun, P. Sassone-Corsi \& A. Means: Calspermin gene transcription is regulated by two cyclic AMP response elements contained in an alternative promoter in the calmodulin kinase IV gene. Mol Cell Biol 15, 561-571, (1995)

15. N.S. Foulkes, F. Schlotter, P. Pévet \& P. SassoneCorsi: Pituitary hormone FSH directs the CREM functional switch during spermatogenesis. Nature 362, 264-267, (1993)

16. L. Monaco, N.S. Foulkes \& P. Sassone-Corsi: Pituitary follicle-stimulating hormone (FSH) induces CREM gene expression in Sertoli cells: Involvement in long-term desensitization of the FSH receptor. Proc Natl Acad Sci USA 92, 10673-10677, (1995)

17. F. Nantel, L. Monaco, N.S. Foulkes, D. Masquillier, M. LeMeur, K. Henriksén, A. Dierich, M. Parvinen \& P. Sassone-Corsi: Spermiogenesis deficiency and germ-cell apoptosis in CREM-mutant mice. Nature 380, 159-162, (1996)

18. J.A. Blendy, K.H. Kaestner, G.F. Weinbauer, E. Nieschlag \& G. Schutz: Severe impairment of spermatogenesis in mice lacking the CREM gene. Nature 380, 162-165, (1996)

19. C.M. Knudson, K.S.K. Tung, W.G. Tourtelotte, G.A.J. Brown \& S.J. Korsmeyer: Bax-deficient mice with lymphoid hyperplasia and male germ cell death. Science 270, 96-99, (1995)

20. D.J. Dix, J.W. Allen, B.W. Collins, C. Mori, N. Nakamura, P. Poorman-Allan, E.H. Goulding \& E.M. Eddy: Targeted gene disruption of HSP70-2 results in failed meiosis, germ cell apoptosis, and male infertility. Proc Natl Acad Sci USA 93, 3264-3268, (1996) 